P40 (continued)

policy of the Department of Army/Navy/Air Force, Department of Defense, or U.S. Government.

Funding: U.S. Army Research Institute of Environmental Medicine

\section{P41 Awareness of Dietary Guidance and Diet Quality of Adults by Race/Ethnicity, What We Eat in America}

Katherine Hoy, EdD, RD, kathy.hoy@ars.usda.gov, Agricultural Research Service, USDA, 10300 Baltimore Avenue, Building 005, Beltsville, MD 20705;

Joseph Goldman, MA, Agricultural Research Service, USDA; Alanna Moshfegh, MS, RD

Objective: To describe awareness of federal dietary guidance, and compare the Healthy Eating Index (HEI) 2010 and its 12 components by level of awareness for adults by race/ethnicity.

Study Design, Setting, Participants: Cross-sectional analyses of data for all adults $19+$ years $(\mathrm{N}=13,022)$ from What We Eat in America, NHANES 2007-2012 for NonHispanic whites (NH-W), Non-Hispanic blacks (NH-B), and Hispanics $(\mathrm{H})$.

Outcome Measures and Analysis: Using paired t-test, the HEI and its components were compared by level of awareness (unaware; aware (only); aware and tried ('tried') of either the Food Guide Pyramid or MyPyramid (FGP/MP) for each race/ethnicity. Comparisons were considered significant at $\mathrm{P}<0.001$.

Results: For NH-W, NH-B, and H, respectively, 57, 47, and $44 \%$ were aware of and 26,24 , and $19 \%$ had looked up/tried the FGP/MP. Mean HEI of those who tried was higher among NH-W (55.2) and NH-B (50.4) than either those aware (NH-W: 50.4; NH-B: 45.9) or unaware (NH-W: 48.5; NH-B: 47.5), with no differences among Hispanics. For 12 HEI components, compared to those either unaware or aware, scores for those who tried were higher for seven components among NH-W, for two components among NH-B, and for none of the components among $\mathrm{H}$. Across all ethnic groups, scores of those who tried were not significantly different from either those aware or unaware for intake of refined grains, total protein foods, fatty acids and sodium.

Conclusions and Implications: These results suggest that the FGP/MP was a resource for individuals trying to improve their diet, particularly Non-Hispanic whites. Future research about MyPlate implementation will be informative.

Funding: USDA, NHANES 2007-2012

\section{P42 Cultural Influences of Diet and Physical Activity by Mexican-Americans with Type 2 Diabetes}

Julie Plasencia, PhD, MS, RD, julieplasencia@uky.edu, University of Kentucky, 212A Funkhouser Building, Lexington, Kentucky 40515; Hector Balcazar, PhD, Charles R. Drew University of Medicine and Science;

Sharon Hoerr, $P h D, R D$, Michigan State University; Maria Lapinski, PhD; Lorraine Weatherspoon, PhD, RD
Objective: The objective of this study was to identify and characterize patient perceived cultural influences on diet and physical activity (PA) behaviors relevant to type 2 diabetes (T2DM) self-management education for MexicanAmerican adults.

Methods: A qualitative synthesis was conducted to identify research studies that met the following criteria: $>50 \%$ of participants included self-identified MexicanAmericans, and findings were related to culturally relevant T2DM self-management diet and/or PA behaviors. Two coders used a content analysis protocol to identify and define cultural elements in the findings of the 19 applicable studies, all guided by the Health Belief Model and Resnicow's theory of cultural sensitivity in public health.

Results: Coders identified and characterized 38 cultural elements. Using Cohen's Kappa coefficient, interrater reliability for cultural elements between the coders ranged from 0.67-1. Cultural elements were categorized as surface or deep level dimensions. The most common surface level elements were food habits $(n=15 / 19)$, food preferences $(n=14 / 19)$, use or acquisition of nutrition knowledge $(11 / 19)$ and healthcare provider preferences (11/19). The top three deep level elements were general health beliefs or attitudes $(n=19 / 19)$, general beliefs or attitudes about foods $(n=17 / 19)$ and family turmoil $(n=14 / 19)$. PA related elements were rare.

Conclusions: Findings highlight patient/client-identified cultural elements relevant to nutrition and PA behaviors for efforts targeting Mexican-Americans with T2DM. These findings have implications for developing and adapting culturally sensitive T2DM self-management education relative to nutrition and PA behaviors.

Funding: College of Agriculture and Natural Resources Research Enhancement Funds

\section{P43 Developing Portion Size Image Contents of Red and Processed Meat for an Intake Analysis Program}

Mi-Hyun Kim,PhD, mhkim1129@ut.ac.kr, Korea National University of Transportation, 61 Daehakro, Jeungpyeong, Chungbuk, South Korea 27909; Jeeyoung Yeon, PhD,

Seowon University; Kyung-Haeng Lee, PhD, Korea National University of Transportation

Objective: To develop portion size of red and processed meat, and their image contents for an intake analysis program that is designed to let people know and control daily red and processed meat consumption.

Theory, Prior Research, Rationale: The International Agency for Research on Cancer (IARC) has classified processed meat as a carcinogen, and red meat as a probable carcinogen. Meat consumption has increased among Koreans and the report by IARC caused people's anxiety about red and processed meat intake. Thus, there is need for developing and implementing an 


\section{P43 (continued)}

intake analysis program that measure red and processed meat intake.

Description: As a first step for developing the program, portion-size analysis of frequently consumed red and processed meat among Koreans was conducted using the 2013-2014 Korea National Health and Nutrition Examination Survey data.

Evaluation: The data was analyzed on 13,460 persons aged 4 and over, and selected food items consumed based on the intake frequency of 30 or more by all participant. A total of 20 varieties of red and processed meat items and 87 varieties of dishes containing red and processed meat were selected. The portion size of red and processed meat and their dishes was set on the basis of the median amount in a single intake by one person. In the second step, photographs of portion size were taken to enhance individuals' estimates on the intake.

Conclusions and Implications: These established portion sizes and photo images of frequently consumed red and processed meat among Koreans can be used in an food intake analysis program based on smart device or Web.

Funding: MFDS

\section{P44 Development of an 'At Risk for Metabolic Syndrome' Score}

Melissa Olfert, DPH, DrPH, RDN, melissa.olfert@mail.wvu. edu, West Virginia University, 333 AG SC Building G28, Morgantown, WV 26506; Oluremi Famodu, MS, RDN, West Virginia University; Rashel Clark, RDN;

Ida Holaskova, PhD; Pamela Murray, MD; Timothy Waander; Chris Molina; Jesse Morrell, PhD, University of New Hampshire

Objective: Develop a score that evaluates individuals 'at risk of metabolic syndrome (metS)' in college-age students using risk factors in addition to the five components of metS (abnormal waist circumference, dyslipidemia, elevated blood pressure, and insulin resistance). Rationale: MetS affects more than $20 \%$ of U.S. adults and was reported in $10 \%$ of male and $3 \%$ of female college students.

Description: Health and nutrition research professionals developed the scoring tool in September 2014. In addition to the five components, the tool considered nutritional status, physical inactivity, ethnicity, and family history. Thirty-seven young adults at West Virginia University (WVU) were evaluated using the 12 point scale.

Evaluation: Correlations were assessed between our tool and two validated tools of disease severity: West Virginia University School of Public Health MetS Severity Score (Gurka et al 2014) and the American Heart Association Score (AHA My Life Check ${ }^{\circledR}$ 2016). Pearson's correlation showed a significant association between our score and WVU's Public Health Z-score $(\mathrm{R}=0.049$ CI $(0.19,0.70)$ $\mathrm{p}=0.0024)$ and the AHA Score $(\mathrm{R}=-0.35$, CI $(-0.60$, -0.03) $\mathrm{p}=0.03)$.

Conclusions and Implications: To our knowledge, no instrument has been developed to assess 'at risk of developing metS'. Our instrument strongly correlates to the most validated tools that currently assess disease severity. Future analysis is being undertaken to evaluate our tool using a larger sample size. Development of this tool helps improve primary prevention intervention strategies on individuals at risk of MetS.

Funding: None

\section{P45 Development of Evaluation Tools to Assess the Effectiveness of a Guided Grocery Store Tour Nutrition Education Program: A Pilot Study}

Rachel Winn, East Carolina University; Jacqueline Sugg, MS, $L D N, R D N$, Pitt County Health Department; Virginia C. Stage, PhD, LDN, RDN, carrawaystagev@ecu. edu, East Carolina University, College of Allied Health Sciences, Health Sciences Building 4310J, Greenville, NC 27834

Objective: Evaluate the effectiveness of a guided grocery store tour to improve Nutrition Knowledge (NK) and Food Selection Behaviors (FSB) among low-income adults in Pitt County, North Carolina.

Study Design, Setting, Participants, Intervention: The study was conducted in collaboration with the Pitt County Health Department and used the national curriculum, Cooking Matters At The Store (CMATS). Local dietitians led each tour and focused on label reading, comparing unit prices, whole grains, and purchasing fruits/vegetables (fresh, frozen, canned). Each tour ended with a "\$10 Challenge" in which participants use knowledge learned to make a healthy meal with a budget. Participants made additional baskets if they did not meet the challenge on the first try.

Outcome Measures and Analysis: NK was evaluated with a 16-item pre/post survey. FSB were evaluated during the $\$ 10$ Challenge by photographing each meal created by participants. Descriptives and Wilcoxin-signed rank tests were used to analyze data.

Results: A total of 28 participants from 10 tours were included in the final sample. Participants were primarily female $(98.6 \%)$ and were Black. NK significantly improved (82.81\% (pre), 89.06\% (post); p-.03). Approximately 69\% of participants met the $\$ 10$ challenge on the first round. No significant differences were observed in calories or macronutrients between the first, and second or third challenge baskets created.

Conclusion and Implications: Overall, evaluation of the CMATS program demonstrated improvement in NK and FSB. Due to high pre-scores, the NK survey questions need revision. Future research will evaluate the program in a larger sample of low-income adults.

Funding: East Carolina University Undergraduate Research \& Creative Activity Award 